\title{
SYNTHESIS AND BIOLOGICAL ACTIVITY OF AMINOMETHYLENE DERIVATIVES OF EMODIN
}

\author{
M. I. Sikharulidze* and V. Yu. Vachnadze
}

UDC 547.99.547.673

The isolation and modification of plant metabolites in order to enhance their biological activity and produce new promising compounds is one of the leading areas of the chemistry of natural compounds.

We synthesized previously [1] aminomethylene derivatives of emodin, 1,6,8-trihydroxy-3-methyl-9,10-anthraquinone, that was isolated from production wastes of the drug ramnil [2]. We first synthesized 7-cytisinylmethyl-1,6,8-trihydroxy3-methyl-9,10-anthraquinone (1) by using the natural secondary amine alkaloid cytisine as the amine component. The source of the cytisine was two species of Leguminosae indigenous to Georgia, Cytisus caucasicus Grossh and Spartium junceum L. (the cytisine content during flowering is $0.08 \%$ of the air-dried aerial part of the raw material).<smiles>[R]Cc1c(O)cc2c(c1O)C(=O)c1c(O)cc(C)cc1C2=O</smiles>

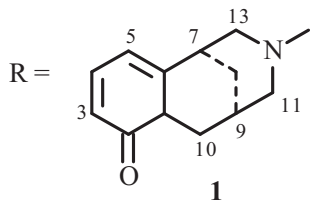<smiles>CN1CCOCC1</smiles>

The Mannich reaction was carried out by the previously described method [1]. The yield of 1 was $92 \%, \mathrm{mp} 254^{\circ} \mathrm{C}$ (dec). IR spectrum $\left(\mathrm{KBr}, v, \mathrm{~cm}^{-1}\right.$ ): 1675 (cytisine $\mathrm{C}=\mathrm{O}$ ), 1650, 1620 (anthraquinone $\mathrm{C}=\mathrm{O}$ ), 1595-1560 (anthr. Ar $\mathrm{C}=\mathrm{C}$ ).

The PMR spectrum of $\mathbf{1}$ showed resonances for anthraquinone and cytisine protons. The lack of a resonance for the anthraquinone C-7 proton indicated that cytisine had been added to it. Resonances of the three $\mathrm{OH}$ protons, which overlapped each other, appeared as a broad resonance at $\delta 10.9 \mathrm{ppm}$.

Resonances of the aromatic anthraquinone and cytisine protons appeared at 7.33-7.53 and 6.22-7.13 ppm; of $\mathrm{CH}_{3}-$ and $\mathrm{N}-\mathrm{CH}_{2}$ protons, at $2.46 \mathrm{ppm}$ as a doublet and at $4.23 \mathrm{ppm}$ as a singlet, respectively.

7-Cytisinylmethyl-1,6,8-trihydroxy-3-methyl-9,10-anthraquinone (1). PMR spectrum [DMSO- $\mathrm{d}_{6}-\mathrm{CCl}_{4}(1: 3)$ $\left.+\mathrm{CF}_{3} \mathrm{COOD}, \delta, \mathrm{ppm}, \mathrm{J} / \mathrm{Hz}\right]: 1.83,1.98\left(2 \mathrm{H}, \mathrm{m}, \mathrm{J}_{\mathrm{a}, \mathrm{b}}=13, \mathrm{H}-8\right), 2.46\left(3 \mathrm{H}, \mathrm{d},{ }^{4} \mathrm{~J}=0.8, \mathrm{CH}_{3}\right), 2.73(1 \mathrm{H}, \mathrm{m}, \mathrm{H}-9), 3.25(1 \mathrm{H}, \mathrm{dd}$, $\left.{ }^{2} \mathrm{~J}=12.7,{ }^{3} \mathrm{~J}=2.2, \mathrm{H}-11\right), 3.31(1 \mathrm{H}, \mathrm{m}, \mathrm{H}-13), 3.34(1 \mathrm{H}, \mathrm{m}, \mathrm{H}-7), 3.46(1 \mathrm{H}, \mathrm{m}, \mathrm{H}-13), 3.62\left(1 \mathrm{H}, \mathrm{d},{ }^{2} \mathrm{~J}=12.7, \mathrm{H}-11\right), 3.79(1 \mathrm{H}$, $\left.\mathrm{dd},{ }^{2} \mathrm{~J}=15.9,{ }^{3} \mathrm{~J}=7.1, \mathrm{H}_{\mathrm{ax}}-10\right), 4.07\left(1 \mathrm{H}, \mathrm{d},{ }^{2} \mathrm{~J}=15.9, \mathrm{H}_{\mathrm{eq}}-10\right), 4.23\left(2 \mathrm{H}, \mathrm{s}, \mathrm{N}-\mathrm{CH}_{2}\right), 6.22\left(1 \mathrm{H}, \mathrm{dd},{ }^{3} \mathrm{~J}=6.9,{ }^{4} \mathrm{~J}=1.3, \mathrm{H}-5\right), 6.35$ $\left(1 \mathrm{H}, \mathrm{dd},{ }^{3} \mathrm{~J}=9.1,{ }^{4} \mathrm{~J}=1.3, \mathrm{H}-3\right), 7.13\left(1 \mathrm{H}, \mathrm{s}, \mathrm{H}-2^{\prime}\right), 7.33\left(1 \mathrm{H}, \mathrm{s}, \mathrm{H}-5^{\prime}\right), 7.35\left(1 \mathrm{H}, \mathrm{dd},{ }^{3} \mathrm{~J}_{1}=9.1,{ }^{3} \mathrm{~J}_{2}=6.9, \mathrm{H}-4\right), 7.53(1 \mathrm{H}, \mathrm{d}$, $\left.{ }^{4} \mathrm{~J}=1.6, \mathrm{H}-4^{\prime}\right), 10.9(3 \mathrm{H}$, br.s, $\mathrm{OH})$.

Biological tests of 1, 7-morpholinomethyl-1,6,8-trihydroxy-3-methylanthraquinone (2), and 7-dimethylaminomethyl1,6,8-trihydroxy-3-methylanthraquinone (3) [1] were performed according to the TAACT program for the discovery of new antituberculosis drugs and the NIAID-AACF program for the discovery of antiviral drugs.

Preliminary screening of 1-3 against Mycobacterium tuberculosis $\mathrm{H}_{37} \mathrm{R}_{\mathrm{v}}$ at concentrations $>10 \mu \mathrm{g} / \mathrm{mL}$ showed low inhibitory activity. These compounds were checked against herpes virus and respiratory viruses. Compound $\mathbf{3}$ exhibited high activity against $\mathrm{HCMV}$, human cytomegalovirus $\left(\mathrm{EC}_{50}=17.5 \mu \mathrm{m}\right.$; toxicity $\left.\mathrm{CC}_{50}>300 \mu \mathrm{m}\right)$. Compound 1 showed pronounced antiviral activity against respiratory viruses. Compound $\mathbf{3}$ is a candidate for further research.

\section{REFERENCES}

1. L. A. Kintsurashvili, M. I. Sikharulidze, V. N. Buyanov, and D. G. Turabelidze, Khim. Prir. Soedin., 722 (1999).

2. $\quad$ A. V. Gotsiridze and E. P. Kemertelidze, Khim. Prir. Soedin., 519 (1971).

I. Kutateladze Institute of Pharmaceutical Chemistry, Tbilisi State Medical University, Tbilisi, 0159, fax: (99532) 520023 , e-mail: docmadona@yahoo.com. Translated from Khimiya Prirodnykh Soedinenii, No. 2, March-April, 2012, p. 273. Original article submitted February 16, 2011. 